\title{
Terminator Device
}

National Cancer Institute

\section{Source}

National Cancer Institute. Terminator Device. NCI Thesaurus. Code C50218.

A device designed to connect to the end of a bus network. 